\title{
Diagnóstico diferencial de infertilidad femenina: Síndrome de Ovario Poliquístico e Hiperplasia Suprarrenal Congénita No Clásica. Revisión Bibliográfica.
}

\section{Differential diagnosis of female infertility: Polycystic ovary syndrome and Non-classical Congenital Adrenal Hyperplasia. A literature review.}

Pág. 195, 205

Recibido: $20-05-2020$

Aceptado: 13-07-2020

María Angélica Montero-Rojas ${ }^{1}$

Daniela Gallardo-Madrigal²

1,2,3,4. Médico General, Trabajador Independiente. San José,Costa Rica.

\section{CONFLICTOS DE INTERÉS}

Ninguno

\section{FINANCIAMIENTO}

La presente investigación no ha recibido ayudas específicas provenientes de agencias del sector público, sector comercial o entidades sin ánimo de lucro.

\section{RESUMEN}

La infertilidad femenina afecta aproximadamente a un $13 \%$ de las mujeres en edad reproductiva globalmente. La causa endocrina más frecuente corresponde al síndrome de ovario poliquístico (SOP) el cual se traslapa fenotípicamente con la hiperplasia suprarrenal congénita no clásica (HSC-NC), por lo que el diagnóstico diferencial entre estos entes se considera mandatorio. El SOP, caracterizado por oligoanovulación, hiperandrogenismo y folículos ováricos en el ultrasonido, se presenta hasta en un $90-95 \%$ de las pacientes que buscan tratamiento para su condición de infertilidad. Por su parte, la HSC-NC es el trastorno de esteroidogénesis genético más común que afecta la fertilidad. Factores como el hiperandrogenismo, desórdenes hormonales, alteraciones anatómicas y el índice de masa corporal juegan un papel en las anormalidades

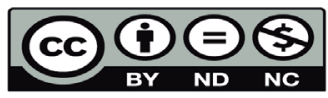


reproductivas que son comunes entre estos dos trastornos endocrinos. El objetivo de esta revisión es discutir las diferencias y similitudes en los mecanismos implicados en el desarrollo de infertilidad de ambos síndromes para realizar un adecuado diagnóstico diferencial.

\section{PALABRAS CLAVE}

Infertilidad femenina, hiperandrogenismo, hiperplasia suprarrenal congénita no clásica, HSC-NC, Síndrome de Ovario Poliquístico, SOP.

\section{ABSTRACT}

Female infertility affects globally approximately $13 \%$ of women of reproductive age. Polycystic ovary syndrome (PCOS) is the most frequent endocrine cause, which phenotypically overlaps with non-classical congenital adrenal hyperplasia (NC-CAH), so the differential diagnosis between these entities is considered mandatory. PCOS, characterized by oligoanovulation, hyperandrogenism, and ovarian follicles on ultrasound, occurs in up to $90-95 \%$ of patients seeking treatment for their infertility condition. Meanwhile, HSC-NC is the most common genetic steroid disorder that affects fertility. Factors such as hyperandrogenism, hormonal anomalies, anatomical alterations and the body mass index play a role in the reproductive abnormalities that are common between these two endocrine disorders. The objective of this review is to discuss the differences and similarities in the mechanisms involved in the development of infertility of both syndromes to make an adequate differential diagnosis.

\section{KEYWORDS}

Female infertility, hyperandrogenism, nonclassical congenital adrenal hyperplasia,
NC-CAH, Polycystic Ovary Syndrome, PCOS.

\section{INTRODUCCIÓN}

La infertilidad o subfertilidad se refiere a la incapacidad de una pareja para establecer un embarazo clínico después de 12 meses de relaciones sexuales regulares sin protección debido a un deterioro de la capacidad de reproducción de una persona, ya sea individualmente o con su pareja $(1,2)$. Se puede clasificar en primaria, en el caso de parejas que nunca han concebido; y secundaria, en parejas que han logrado la concepción en algún momento(2). Se estima que en países en desarrollo una de cada cuatro parejas en edad reproductiva padecen de infertilidad(1). Aproximadamente un $13 \%$ de las mujeres globalmente padecen de infertilidad, siendo la secundaria la forma más común $(1,2)$.

La infertilidad femenina se debe en su vasta mayoría a una disfunción ovulatoria (2), cuya causa endocrina más frecuente en mujeres en edad reproductiva corresponde al síndrome de ovario poliquístico $(\mathrm{SOP})(1,3,4)$. Debido al gran parecido fenotípico de este síndrome con la hiperplasia suprarrenal congénita no clásica (HSC-NC), el diagnóstico diferencial entre estos dos trastornos es mandatorio en el contexto de infertilidad femenina (5). Por tanto, el objetivo de esta revisión es discutir las diferencias y similitudes en los mecanismos implicados en el desarrollo de infertilidad de ambos síndromes para realizar un adecuado diagnóstico diferencial.

EI SOP se caracteriza por oligoovulación y/o anovulación, signos químicos o bioquímicos de hiperandrogenismo y la visualización de folículos ováricos en ultrasonido; características que a su vez forman parte de los criterios de 
Rotterdam (6-8). Este síndrome corresponde a la principal causa de hiperandrogenismo y oligoanovulación que asocia infertilidad y desórdenes metabólicos, dentro de los cuales predomina la resistencia a la insulina, intolerancia a la glucosa y diabetes mellitus tipo 2 . Además, conlleva implicaciones de por vida al aumentar el riesgo de infertilidad, hiperplasia endometrial, desórdenes metabólicos, carcinoma endometrial y enfermedad cardiovascular $(4,6,9)$.

EI SOP abarca múltiples factores reproductivos y metabólicos, y globalmente afecta cerca de un $4-18 \%$ de las mujeres. Además, la fertilidad de estas pacientes puede verse afectada de forma negativa por el estado individual de sobrepeso, hiperandrogenismo y concentraciones elevadas de hormona luteinizante $(3,4,10)$.

La hiperplasia suprarrenal congénita (HSC) es un trastorno autosómico recesivo, causado por una esteroidogénesis defectuosa en la corteza suprarrenal. Más del $90 \%$ de los casos son causados por la deficiencia de la enzima 21-hidroxilasa(11). La anormalidad bioquímica más característica es la elevación de 17-hidroxiprogesterona (17-OHP), el sustrato principal de la 21 hidroxilasa; lo que desplaza el eje hacia la producción de androstenediona y testosterona (12). La HSC$\mathrm{NC}$ es una forma leve de HSC, ya que presenta una actividad enzimática residual del 20 al $60 \%$ $(5,13)$. Corresponde a uno de los trastornos autosómicos recesivos más comunes y es el trastorno esteroidal genético más frecuente $(13,14)$.

Se estima que la prevalencia de HSC-NC es de 1 en 600, dependiendo de la etnia; $(11,12)$; y del $1-10 \%$ en mujeres con hiperandrogenismo (15). Sin embargo, existe una subestimación considerable debido a que el cribado de recién nacidos no revela una 17-OHP elevada, además de que esta condición no cursa con ambigüedad genital al nacimiento (13). Este desorden se presenta con varios grados de virilización postnatal de acuerdo con la gravedad del defecto; puede ser asintomática (12); con manifestaciones en la adolescencia y en mujeres jóvenes como hiperandrogenismo, oligoovulación, pubarca precoz, cuadro clínico similar al SOP e infertilidad; o bien, en casos más graves, desde la infancia con síntomas como pubarca prematura, acné y edad ósea acelerada (13).

\section{INFERTILIDAD FEMENINA EN SOP E HSC-NC}

EI SOP es la principal causa de infertilidad anovulatoria, hasta un $90-95 \%$ de las pacientes que buscan tratamiento para su condición de infertilidad lo presentan. Una de las implicaciones reproductivas más importantes es el riesgo de pérdida gestacional temprana; además de que usualmente les toma más tiempo lograr el embarazo $(4,16,17)$. De manera general se ve caracterizado por anovulación fisiológica, elevación de la hormona luteinizante (LH), hiperandrogenismo y anormalidades en la frecuencia de pulsaciones de hormona liberadora de gonadotropina $(\mathrm{GnRH})$. Estas alteraciones bioquímicas se manifiestan como acné, hirsutismo, entre otros $(6,7,9)$.

Por su parte, la HSC es el trastorno de esteroidogénesis genético más común que afecta la fertilidad. Las hormonas esteroideas suprarrenales son esenciales para el desarrollo sexual normal y la función reproductiva. Esta infertilidad se ha explicado por alteraciones del eje hipotálamo-hipófisis-ovario ( $\mathrm{HHO})$, desencadenado por andrógenos elevados, progesterona aumentada, expresión de $5 a$-reductasa en el ovario e incluso como un

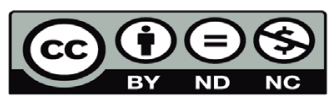


efecto glucocorticoide directo (11).

Existe una correlación entre la gravedad del defecto enzimático y la tasa de embarazo espontáneo y fertilidad en mujeres con HSC. La verdadera tasa de fertilidad en mujeres con HSCNC es difícil de obtener debido al subestimación de esta condición (18). Se ha reportado que hasta en el $13 \%$ de los casos de NC-CAH la infertilidad es el síntoma de presentación (13). Más del $30-50 \%$ de los pacientes con $\mathrm{NCAH}$ muestran disfunción ovulatoria y menstrual (15).

Las mujeres con HSC-NC tienen menos posibilidades de presentar anovulación; mientras que en el SOP está representa el $70-90 \%$ de los trastornos ovulatorios. Alrededor del $50 \%$ de las mujeres con SOP presentan infertilidad primaria y el $25 \%$ infertilidad secundaria. En cambio, solo el $17 \%$ de las mujeres con HSC$\mathrm{NC}$ tienen irregularidades menstruales. Además, la subfertilidad en mujeres con HSC-NC es más leve y muchas mujeres llegan a concebir espontáneamente (5). Aunado a la infertilidad, estos padecimientos se relacionan con otras condiciones que complican el embarazo. Las mujeres con SOP poseen un alto riesgo de parto prematuro, preeclampsia y diabetes gestacional durante el embarazo. La tasa de aborto espontáneo es $20-40 \%$ más alta que las mujeres sanas. Por su parte, en pacientes con HSC-NC existe un riesgo significativo de aborto espontáneo, aproximadamente del 25\% (5).

\section{Mecanismos implicados en la infertilidad}

\section{Hiperandrogenismo}

La etiología pura de la hiperandrogenemia en el SOP se desconoce, se sabe que factores ambientales pueden influir. Sin embargo, la literatura menciona que este es causado primariamente por un desequilibrio o defecto celular tecal y disfunción del eje $\mathrm{HHO}$, este último manifestándose con una adrenarca prematura como signo temprano $(6,9)$. El mecanismo de la anovulación no se le atribuye a los andrógenos dado que se encuentran altos niveles de andrógenos en portadoras de SOP sin que esto sea la causa de la anovulación(19). Existe evidencia que al menos un tercio de las pacientes presentan disfunción adrenocortical en la formación de andrógenos, con un exceso de sulfato de dihidroepiandrosterona (DHEAS). Sin embargo, el rol de los andrógenos adrenales como causante de infertilidad en el SOP sigue sin ser confirmado. Por el contrario, el hiperandrogenismo es el factor más destacado que contribuye en la infertilidad de mujeres con HSC-NC (18).

En cuanto a la HSC-NC, los niveles séricos de DHEAS son generalmente normales, mientras que la androstenediona, la testosterona y la dihidrotestosterona (DHT) están elevados, pero son similares a los niveles encontrados en pacientes con SOP (15). El hiperandrogenismo en la HSC-NC resulta de la hiperactividad suprarrenal, de una mayor conversión periférica de metabolitos esteroides a andrógenos y del aumento de la secreción de andrógenos ováricos (15). La elevación androgénica produce alteraciones del eje $\mathrm{HHO}$, altera las vías normales de retroalimentación central e interfiere con la generación del pulso de la hormona liberadora de gonadotropina $(\mathrm{GnRH})$, lo cual dificulta la ovulación (18). Sin embargo, existe discusión sobre el mecanismo exacto por el cual los andrógenos alteran el eje $\mathrm{HHO}$. Mujeres con HSC-NC que reciben tratamiento con esteroides normalizan los niveles de $\mathrm{LH}$ y la capacidad de respuesta de LH a los pulsos 
de GnRH. No obstante, cuando se ha utilizado andrógenos como tratamiento en mujeres con SOP no se ha alterado la secreción basal de $\mathrm{LH}$, aunque las dosis altas de andrógenos sí redujeron ligeramente la concentración de la $\mathrm{FSH}(13)$.

También se ha propuesto que los andrógenos poseen un efecto directo sobre el ovario, pues se ha visto que afectan la foliculogénesis (18). Se ha demostrado que los andrógenos suprarrenales elevados inhiben la actividad de la aromatasa de las células de la granulosa (13). Los andrógenos actúan como moduladores complejos de la esteroidogénesis ovárica debido a la inhibición de la formación de receptores de $\mathrm{LH}$, estimulados por la $\mathrm{FSH}$, en las células de la granulosa, cuyos efectos varían de acuerdo al alcance y la duración de la exposición a los andrógenos $(13,18)$.

\section{Otros factores hormonales}

El SOP es el desorden hormonal más común, causante de infertilidad por anovulación en un $80 \%$ de las mujeres $(7,9)$. La infertilidad puede ser parcialmente explicada por la exposición a factores ambientales y desbalances hormonales. Como previamente se describió, la clínica se caracteriza por oligoanovulación, la cual puede presentar diferentes patrones clínicos y anormalidades en el ciclo menstrual $(7,10,19)$.

Se sugiere que el arresto en el crecimiento de folículos antrales y la anovulación son consecuencia de un desarreglo temprano en el desarrollo del folículo, e inclusive pueden influir factores paracrinos a nivel de líquido folicular que contribuyen a este arresto $(19,20)$. Se ha demostrado que pacientes portadoras de SOP con ciclos anovulatorios poseen células de la granulosa con una hiperrespuesta a la FSH en términos de producción de estradiol y tienden a presentar una respuesta inapropiada a $\mathrm{LH}$, lo que se cree provoca una diferenciación de la granulosa que resulta en un arresto prematuro en el crecimiento folicular (19).

Las pacientes portadoras de SOP presentan niveles elevados de $\mathrm{LH}$ en contraste con $\mathrm{FSH}$ los cuales se encuentran disminuidos $(7,8)$. El aumento de los niveles de LH estimulan la secreción de andrógenos por parte de las células de la teca, lo que resulta en la hiperandrogenemia que caracteriza el síndrome. Otros factores como el aumento de insulina circulante, desregulación de factores moduladores de reclutamiento y crecimiento de folículos, proteínas y citoquinas pueden influir en el desarrollo folicular anormal (9).

En cuanto a la HSC-NC, la anovulación es la principal causa de subfertilidad en estas mujeres, lo cual es más evidente por la respuesta positiva a la inducción de la ovulación (15). La evidencia indica que existe una hipersecreción de LH, similar al SOP. El exceso de andrógenos deteriora la sensibilidad del hipotálamo a la progesterona, lo que aumenta la frecuencia de los pulsos de la $\mathrm{GnRH}$ y resulta en una secreción preferencial de la LH. La hipersecreción de LH aumenta la producción de andrógenos ováricos y se potencian los efectos de los andrógenos suprarrenales (11). En comparación con los controles, las mujeres con HSC-NC han aumentado la amplitud del pulso de LH pero a intervalos normales (18).

Por otro lado, la aromatización del exceso de andrógenos aumenta los niveles de estrógenos circulantes, los cuales pueden suprimir el eje $\mathrm{HHO}$, conducir a la anovulación y a ciclos menstruales irregulares (18). Asimismo, las

\section{(9) $(1) \Theta \theta$}


mujeres con HSC-NC cursan con niveles elevados de progesterona consistentemente a lo largo del ciclo, en contraste con el patrón bifásico normal de pacientes sanas. Esto altera la calidad de los ovocitos y la frecuencia del pulso de la $\mathrm{GnRH}$, lo que puede impedir la ovulación e implantación. Tanto la progesterona como la 17-OHP pueden convertirse en andrógenos más potentes como la DHT (15). También, la progesterona actúa como una forma de anticoncepción ya que altera la motilidad tubárica, espesa el moco cervical, y disminuye la receptividad endometrial $(11,18)$.

\section{Alteraciones anatómicas}

Estudios han demostrado la relación entre anormalidades uterinas y el SOP (16).Durante el desarrollo embrionario del feto femenino los conductos mullerianos están separados por un septo, el cual origina un útero inicialmente septado. Sin embargo, este septo sufre lisis mediante múltiples mecanismos de apoptosis.

Las anormalidades en la fusión o desaparición de estos conductos mullerianos puede resultar en una variedad de anomalías. A pesar de que el útero y los ovarios tienen origen embrionario distinto, la relación antes mencionada podría reflejar un desarrollo común de etiopatogénesis entre el útero y el ovario, que requiere ser investigado con más profundidad.

Los estudios acusan a la genética como principal responsable de estas anomalías; pues tres cuartas partes de las pacientes que cursan con alteraciones estructurales uterinas presentan SOP, lo que aumenta el número de abortos, y con ello afecta la tasa de fertilidad (16).
Por su parte, dado que en la HSC-NC la deficiencia enzimática es leve, la elevación de los andrógenos no se traduce en el desarrollo de anormalidades genitales intra útero (18). Sin embargo, un porcentaje de pacientes con HSC-NC cursan con tumores ováricos de restos suprarrenales, los cuales se cree que son un contribuyente significativo en la génesis de la subfertilidad (18). Además, estudios han encontraron ovarios agrandados en el $44 \%$ de las mujeres adultas con HSC-NC estudiadas.

Esto se debe a que la acción de los andrógenos conduce al engrosamiento de la cápsula ovárica, un aumento general en el tamaño del ovario y una expansión en el número de folículos preantrales (13).

\subsection{Morfología ovárica}

El ultrasonido transvaginal (TVS) es una herramienta para el diagnóstico de SOP, pues evidencia una morfología poliquística ovárica y oligoovulación en ausencia de otros procesos causantes. Los criterios de Rotterdam establecen la definición de ovario poliquístico como la presencia de más de 12 folículos de 2-9mm de diámetro apreciados como quistes en la corteza ovárica, al menos en un ovario y/o un volumen ovárico mayor a $10 \mathrm{~cm} 3(10 \mathrm{ml})(4,7)$.

Los ovarios poliquísticos en la ecografía son más comunes en el SOP que en la HSC-NC (70\% frente a $40 \%$, respectivamente). Por lo tanto, la apariencia ecográfica de ovarios poliquísticos no es una característica específica en el SOP (5). La asociación con anovulación crónica y ciclos menstruales irregulares en HSC puede disminuir aún más la fecundidad de estas mujeres (11). 


\section{4. Índice de masa corporal}

El rol del índice de masa corporal (IMC) abarca los demás factores previamente mencionados, dado que los impactos de la combinación de obesidad y desórdenes alimenticios exacerban la morbilidad reproductiva, metabólica y psicológica (7).

La grasa corporal ejerce un papel como reguladora en la reproducción. Grandes cantidades de células grasas producen grandes cantidades de estrógenos, lo cual ejerce un efecto anticonceptivo. No obstante, la falta de tejido adiposo provoca un déficit de estrógenos, lo cual interrumpe el ciclo menstrual y la ovulación (21).

Aproximadamente un $40-60 \%$ de las pacientes portadoras de SOP presentan obesidad $(6,9)$, la cual forma parte del síndrome metabólico característico de este padecimiento. Un $27.2 \%$ de las pacientes portadoras de SOP asocian un síndrome metabólico que cursa con obesidad central, resistencia a la insulina y dislipidemia $(8,22,23)$

Estudios evidencian que la severidad del hirsutismo y de la disfunción menstrual correlaciona directamente con el grado de resistencia a la insulina (9). Adicionalmente, la hiperinsulinemia contribuye a la desregulación de la $\mathrm{LH}$, e incrementa la producción de andrógenos, la anovulación y el riesgo metabólico $(6,9)$. Pacientes con SOP y síndrome metabólico tienen una elevada lipólisis en el fluido folicular, altos niveles de colesterol y triglicéridos, con una disminución de lipoproteínas de alta densidad (HDL, por sus siglas en inglés); lo que se cree influye en el desarrollo embrionario y altera el metabolismo de lípidos y glucosa. Además, produce una disfunción esteroidogénica de óvulos y de la potencial receptividad del endometrio, por lo que estas pacientes presentan un impacto negativo en fecundidad dada la pobre respuesta a estimulación ovárica y a la fertilización in vitro (IVF) (23).

El impacto del IMC en fertilidad se respalda con estudios que evidencian que la pérdida de peso puede tener un efecto positivo al propiciar un cambio en la función ovulatoria, además de mejorar el estado metabólico $(9,19)$.

Un estudio transversal prospectivo evaluó a 103 pacientes jóvenes con delgadez u obesidad desde la infancia, quienes se sometieron a análisis clínicos y hormonales completos. Se estudió su función menstrual, la prevalencia de trastornos reproductivos y las correlaciones de los cambios hormonales respectivos con el IMC. Se estableció que el IMC se correlaciona negativamente con la $\mathrm{FSH}$ y la globulina transportadora de hormonas sexuales (SHBG) y se correlaciona positivamente con la testosterona libre (TL) y testosterona total (TT).

Se obtuvo que el SOP y el síndrome metabólico fueron los padecimientos más frecuentes en mujeres con sobrepeso y obesidad, con evidencia que sugiere que la obesidad infantil contribuye al desarrollo del SOP adolescente.

La HSC-NC y la disfunción ovárica prevalecieron en mujeres con bajo peso. Por otro lado, el IMC también se ha relacionado con el aumento del riesgo de abortos espontáneos, tanto en mujeres

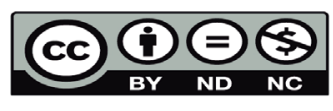


con bajo peso como en mujeres con sobrepeso $u$ obesas (21).

\section{Diagnóstico diferencial de SOP e HSC-NC en el contexto de infertilidad}

Para la realización de un diagnóstico certero que permita la toma de decisiones terapéuticas adecuadas para la fertilidad de la paciente se deben tomar en cuenta desórdenes que simulan estos padecimientos además de orientar el diagnóstico con exámenes de laboratorio (figura 1).

La definición de PCOS ha sido ampliamente discutida por lo que se establecieron los criterios de Rotterdam para la simplificación de su diagnóstico.

La medición de hormonas circulantes en SOP tiene tres propósitos principales, demostrar el hiperandrogenismo, confirmar y demostrar la disfunción ovárica y excluir desórdenes similares (9), principalmente los trastornos tiroideos, hiperprolactinemia e HSC-NC, mediante la medición de la hormona estimulante de tiroides, prolactina y la 17-hidroxiprogesterona, respectivamente.

Asimismo, el Colegio Americano de Ginecólogos y Obstetras (ACOG) recomienda el cribado de la HSC-NC con 17-hidroxiprogesterona en aquellas mujeres con elevado riesgo para esta, dado que el diagnóstico temprano y tratamiento corticosteroide podría mejorar el perfil reproductivo. Sin embargo, todas las mujeres con signos claros de hiperandrogenismo, síntomas y quejas, independientemente del grado, deben ser estudiadas por esta condición (9).
Para el cribado de HSC-NC se utiliza un corte basal de 17 OHP de $2 \mathrm{ng} / \mathrm{ml}$ ( $6 \mathrm{nmol} / \mathrm{l})$, menos de este valor el diagnóstico de HSC-NC es poco probable (15).

El diagnóstico de la HSC-NC se basa en el hallazgo de concentraciones séricas de 17-OHP superiores a $10 \mathrm{ng} / \mathrm{ml}(30 \mathrm{nmol} / \mathrm{L}$ ) en condiciones basales, temprano en la mañana durante la fase folicular del ciclo menstrual, o después de la estimulación con hormona adrenocorticotrópica (ACTH). La 17-OHP basal aumenta durante la fase lútea del ciclo menstrual.

Por lo tanto, la muestra debe obtenerse dentro de los 10 primeros días después del comienzo de la menstruación o en cualquier momento en que la paciente esté en amenorrea (5). Cuando se obtiene un aumento del resultado basal de 17-OHP inferior a $10 \mathrm{ng} / \mathrm{ml}$ debe confirmarse mediante el hallazgo de 17-OHP por encima de $10 \mathrm{ng} / \mathrm{ml}$ después de la estimulación con 250 mcg de ACTH. $(5,15)$.

Debido a que alrededor del $20 \%$ de las pacientes con SOP poseen valores elevados de 17-OHP (5), de estar disponible, se debe realizar la confirmación diagnóstica con el genotipo del CYP21A2. Asimismo, respuestas normales de 17-OHP basales y estimuladas por ACTH no excluyen un estado portador para mutaciones (15). 
Figura 1. Algoritmo para el diagnóstico diferencial de infertilidad femenina. Fuente: Elaboración propia con base en las referencias $(5,15)$.

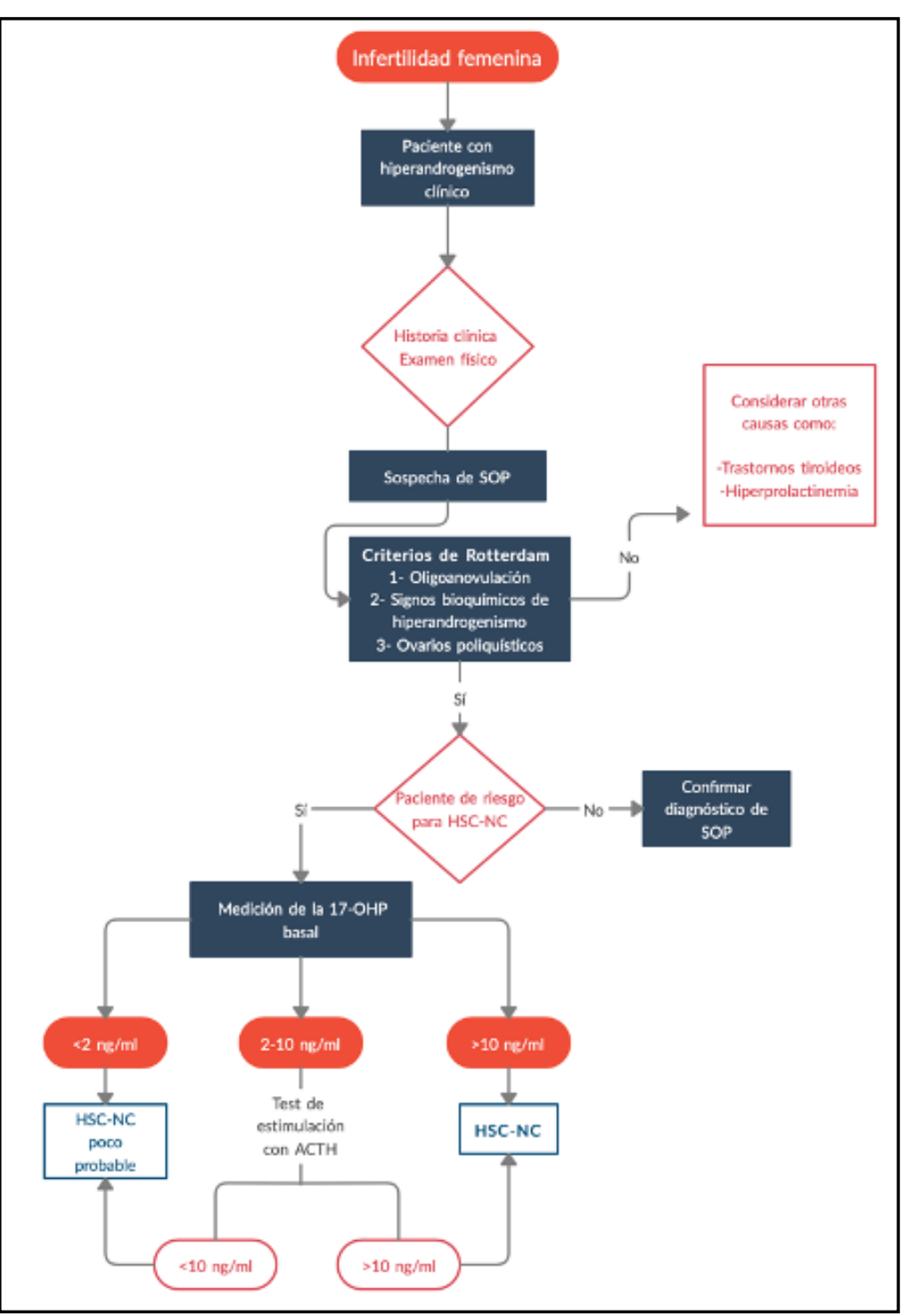

\section{Conclusiones}

El diagnóstico diferencial entre el SOP y la HSC-NC exige una comprensión amplia de los mecanismos fisiopatológicos que los caracterizan. El rol de los andrógenos adrenales como causantes de infertilidad en el
SOP aun no se ha confirmado; mientras que en la HSC-NC se ha demostrado que la elevación androgénica produce alteraciones del eje $\mathrm{HHO}$ e interfiere con los pulsos de la $\mathrm{GnRH}$. Otros factores hormonales se han vinculado con una foliculogénesis anormal, en el caso del SOP debido a una hiperrespuesta a la FSH y una respuesta inapropiada a $\mathrm{LH}$; y en cuanto a la HSC-NC, producto de una secreción preferencial de LH y los niveles elevados de estrógenos y progesterona.

El SOP se ha relacionado con alteraciones anatómicas uterinas, en contraposición con la HSC-NC, que no cursa con anormalidades genitales intra útero. Ambos síndromes pueden cursar con una morfología de ovario poliquístico por lo que este hallazgo no es útil para realizar una diferenciación certera. Asimismo, en ambas patologías el IMC desempeña un papel como regulador en la reproducción, relacionado con una disfunción esteroidogénica de los óvulos y la asociación con hiperinsulinemia.

Se recomienda el uso de técnicas auxiliares de laboratorio para sustentar el diagnóstico entre estos entes. Al sospechar de HSC-NC se utiliza un corte basal de 17 OHP durante los 10 primeros días después del comienzo de la menstruación o al presentarse amenorrea en la paciente. El corte basal de esta prueba se estandarizó en $2 \mathrm{ng} / \mathrm{ml}$, un valor menor a este convierte el diagnóstico de HSC-NC en poco probable. 


\section{BILBIOGRAFÍA}

1. Vander Borght $M$, Wyns $C$. Fertility and infertility: Definition and epidemiology. Clin Biochem. 2018;62(February):2-10.

2. DeCherney, A., Olivares Bari, S. and Padilla Sierra, G., 2014. Diagnóstico Y Tratamiento Ginecoobstétricos. 11th ed. México: McGrawHill Education, pp.879-889.

3. Costello MF, Misso ML, Wong J, Hart R, Rombauts L, Melder A, et al. The treatment of infertility in polycystic ovary syndrome: A brief update. Aust New Zeal J Obstet Gynaecol. 2012;52(4):400-3.

4. Hanson B, Johnstone E, Dorais J, Silver B, Peterson CM, Hotaling J. Female infertility, infertility-associated diagnoses, and comorbidities: a review. J Assist Reprod Genet [Internet]. 2017;34(2):167-77. Available from: http://dx.doi.org/10.1007/s10815-016-0836-8

5. Papadakis G, Kandaraki EA, Tseniklidi E, Papalou O, Diamanti-Kandarakis E. Polycystic ovary syndrome and NC-CAH: Distinct characteristics and common findings. A systematic review. Front Endocrinol (Lausanne). 2019;10(JUN).

6. Hachey LM, Kroger-Jarvis M, Pavlik-Maus T, Leach R. Clinical Implications of Polycystic Ovary Syndrome in Adolescents. Nurs Womens Health [Internet]. 2020;24(2):115-26. Available from: https://doi.org/10.1016/j.nwh.2020.01.011

7. Balen AH, Morley LC, Misso M, Franks S, Legro RS, Wijeyaratne CN, et al. The management of anovulatory infertility in women with polycystic ovary syndrome: An analysis of the evidence to support the development of global WHO guidance. Hum Reprod Update. 2016;22(6):687-708.

8. Dennett CC, Simon J. The role of polycystic ovary syndrome in reproductive and metabolic health: Overview and approaches for treatment. Diabetes Spectr. 2015;28(2):116-20.

9. Azziz R. Reproductive endocrinology and infertility: Clinical expert series polycystic ovary syndrome. Obstet Gynecol. 2018;132(2):321-36.

10. Joham AE, Teede HJ, Ranasinha S, Zoungas S, Boyle J. Prevalence of infertility and use of fertility treatment in women with polycystic ovary syndrome: Data from a large community-based cohort study. J Women's Heal. 2015;24(4):299-307.

11. Pereira N, Lin-su K. Reproductive Function and Fertility in Women with Congenital Adrenal Hyperplasia. 2018;(August):101-7.

12. Ayalon-Dangur I, Segev-Becker A, Ayalon I, Eyal O, Israel S, Weintrob N. The many faces of non-classic congenital adrenal hyperplasia. Vol. 19, Israel Medical Association Journal. 2017. p. 317-22. 
13. New MI, Ghizzoni L, Meyer-Bahlburg H, Khattab A, Reichman D, Rosenwaks Z. Fertility in patients with nonclassical congenital adrenal hyperplasia. Fertil Steril [Internet]. 2019;111(1):1320. Available from: https://doi.org/10.1016/j.fertnstert.2018.11.023

14. Livadas $\mathrm{S}$, Bothou $\mathrm{C}$. Management of the female with non-classical congenital adrenal hyperplasia (NCCAH): A patient-oriented approach. Front Endocrinol (Lausanne). 2019;10(JUN):111.

15. Carmina E, Dewailly D, Escobar-Morreale HF, KelestimurF, Moran C, Oberfield S, et al. Non-classic congenital adrenal hyperplasia due to 21-hydroxylase deficiency revisited: An update with a special focus on adolescent and adult women. Hum Reprod Update. 2017;23(5):580-99.

16. Saleh HA, Shawky Moiety FM. Polycystic ovarian syndrome and congenital uterine anomalies: The hidden common player. Arch Gynecol Obstet. 2014;290(2):355-60.

17. Melo AS, Ferriani RA, Navarro PA. Treatment of infertility in women with polycystic ovary syndrome: Approach to clinical practice. Clinics. 2015;70(11):765-9.

18. Lekarev O, Lin-Su K, Vogiatzi M. Infertility and Reproductive Function in Patients with Congenital Adrenal Hyperplasia. Endocrinology and Metabolism Clinics of North America. 2015;44(4):705-722.

19. Carmina E. Reproductive System Outcome Among Patients with Polycystic Ovarian Syndrome. Endocrinol Metab Clin North Am. 2015;44(4):787-97.

20. Bongrani, A., Mellouk, N., Rame, C., Cornuau, M., Guérif, F., Froment, P. and Dupont, J., 2019. Ovarian Expression of Adipokines in Polycystic Ovary Syndrome: A Role for Chemerin, Omentin, and Apelin in Follicular Growth Arrest and Ovulatory Dysfunction?. International Journal of Molecular Sciences, 20(15), p.3778.

21. Kristesashvili J, Chikvaidze N, Gegechkori M. Types of reproductive disorders in underweight and overweight young females and correlations of respective hormonal changes with BMI. Vol. 36, Giornale Italiano di Ostetricia e Ginecologia. 2014. p. 521-3.

22. Huang-Doran I, Franks S. Genetic Rodent Models of Obesity-Associated Ovarian Dysfunction and Subfertility: Insights into Polycystic Ovary Syndrome. Front Endocrinol (Lausanne). 2016;7(June).

23. He Y, Lu Y, Zhu Q, Wang Y, Lindheim SR, Qi J, et al. Influence of metabolic syndrome on female fertility and in vitro fertilization outcomes in PCOS women. Am J Obstet Gynecol [Internet]. 2019;221(2):138.e1-138.e12. Available from: https://doi.org/10.1016/j.ajog.2019.03.011

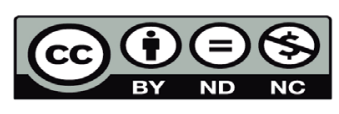

